\title{
Impact of Different Ways of Fatigue on The Shooting of Basketball Players in College
}

\author{
ZhenYu Qiu, YiBao Tong \\ TianJin University Renai College, TianJin 300000,China
}

Keywords: Fatigue; Shooting; Colleges; Basketball; Hit rate

\begin{abstract}
This paper tends to make a research with 24 players from high-level basketball teams of Tianjin University and China Civil Aviation University as research objects. Having divided them into 3 groups which respectively received 1500-meter run, 2 minutes continuous vertical jumps and 1 minute pushups, the author tends to establish 3 models of fatigue on them and explore the influence of each model on penalty shot, two-point shooting and three-point shooting, in order to provide data for the training and relevant research of college basketball.
\end{abstract}

\section{Introduction}

Modern basketball competition is becoming more and more intense, in many factors that affect the outcome of the game, the shooting is undoubtedly the key factor decisive. The displacement of the ball will determine the shot when shots hit rate, but the ball displacement situation, depending on the height of the movement of body parts and jump action, size and position during take-off decisions; shot point is affected by gravity height and speed change from its strength from the torso, legs, arms and wrists, joint production; the last ball and ball spin sphere itself except by the impact, the force from the angular acceleration change various parts of the torso, shoulders, elbows, wrists and fingers and other joints. Therefore, the body condition of athletes is the most important factor affecting the rate of shooting. When athletes fatigue due to fatigue or injury,Muscle will not be able to maintain a certain power output, it is bound to affect the act of shooting, thereby affecting the hit rate.Intense competition for the players is a big physical challenge, how to decline in physical fitness, in the case of physical fatigue, can still make the need for multiple joint involvement of shooting action to achieve the perfect coordination of the players and coaches are badly in need of answers, trying to understand the impact of fatigue on the shooting caused, for coaches and players and Related research officers.

\section{Subjects}

Choose Tianjin University and Civil Aviation University of China high level basketball players a total of 24, with an average age of $20.1 \pm 1.3$ years; height was $186.7 \pm 6.8 \mathrm{~cm}$; weight was $73.6 \pm$ $9.7 \mathrm{~kg}$; ball age of $8.4 \pm 2.36$ years.

2 Research Methods

\subsection{Interview}

Many phone calls or interviews to domestic experts and scholars on the Middle School Advisory Fatigue way of shooting.

\subsection{Test experimental method}

\subsection{1 grouping and pretest.}

24 subjects were randomly divided into A, B, C three groups, before tests 3.3 kinds shooting mode shooting test, namely: the free-throw (the midpoint of the free throw line), two points jumper (the midpoint of the free throw line and the third line median midpoint), three-point set shot (three-point line in the middle), three shots per person, each way to vote 20 times in a row, recording three hits apiece shooting mode situation. 


\subsubsection{Fatigue Model.}

A group of players with personal best $70 \%$ of the time ran 1500 meters; Group B members to $80 \%$ personal best vertical vertical jump height as the standard height point, continued to jump up to two minutes more than the standard height; group $\mathrm{C}$ with individual players $75 \%$ of maximum strength of the resistance to push way means test, one minute continuous uninterrupted motion. Three kinds of motion alternately, each rest for 5 minutes. Three times a week, every other day, a total of four weeks.

\subsubsection{Posttest.}

Third group of players after training fatigue, subjective feel immediately after fatigue testing 3.3 kinds shooting mode shooting, with the former test, recording the hit rate situation.

\section{Results and discussion}

3.1 Compare the difference between the 1500 meter running and the three shooting methods in the test before and after the test

Table 11500 meter running test before and after the T value test table

\begin{tabular}{lccc}
\hline $\begin{array}{l}\text { Number of people } \\
\mathrm{n}=24\end{array}$ & $\begin{array}{c}\text { Before testing } \\
\mathrm{M}(\mathrm{S} . \mathrm{D})\end{array}$ & $\begin{array}{r}\text { After testing } \\
\text { M(S.D) }\end{array}$ & The value of T \\
\hline Free throw & $74.2 \pm 8.5$ & $75.8 \pm 7.3$ & -0.58 \\
Half shot & $57.8 \pm 14.7$ & $52.3 \pm 14.2$ & 2.687 \\
Three-point shooting & $38.4 \pm 18.6$ & $40.1 \pm 16.3$ & -1.86 \\
\hline
\end{tabular}

Note: $\mathrm{P}<0.05$ for significant differences

From table 1 shows that two points before the test scores than the test results, the test to achieve significant level, $\mathrm{P}<0.05$; the free throw and three points shooting did not reach a significant difference, and are better than the post test results.The main reasons are: first, 1500 meters running belong to endurance projects, leading to fatigue in the way of heart and lung fatigue, lower limb muscle effect, but the impact on the upper limb muscle is relatively small.Therefore, for the fixed point shooting in the free throw and in situ three points, its hit rate is not affected.The two point ball shooting distance is the most difficult to judge the distance of the players, and the body's consistency and strength of the control of the ball has a greater difficulty. When players in situ free throws, not the pace of mobile or jumps, the hit rate is less affected; and second shot is to take a jump shooting, the consistency of the body and force control is more difficult, fatigue hit rate will naturally decrease; one-third of the ball is final testing project. At this time, the player's physical ability may partially restored, or fatigue than just finished testing the fatigue degree and the posttest scores but was superior to that of the previous test.

3.2 Comparison of two minutes difference for continuous vertical jump run three hits in shooting mode before and after the test

Table 2 Two minutes of continuous vertical jump test before and after the T value test table

\begin{tabular}{lccl}
\hline $\begin{array}{c}\text { Number of people } \\
\mathrm{n}=24\end{array}$ & $\begin{array}{c}\text { Before testing } \\
\text { M(S.D) }\end{array}$ & $\begin{array}{r}\text { After testing } \\
\text { M(S.D) }\end{array}$ & The value of $\mathrm{T}$ \\
\hline Free throw & $71.2 \pm 11.5$ & $73.7 \pm 12.3$ & 5.63 \\
Half shot & $56.8 \pm 13.5$ & $59.3 \pm 14.7$ & 3.246 \\
Three-point shooting & $34.4 \pm 13.5$ & $36.8 \pm 15.3$ & 2.49 \\
\hline
\end{tabular}

Note: $\mathrm{P}<0.05$ for significant differences

From table 2, we can see that after two minutes of continuous vertical jump test results in three kinds of shooting are no significant difference, and the test results are better than the previous test.Continuous vertical jumping is under the waist muscle and joint strength increased training, no to fixed-point shooting body coordination has seriously affected, continuous jump shooting 
movement and shooting similar. Therefore, it is possible will improve shooting skills and hit rate.In the act of shooting three indicators that shot height, speed and range, height and speed depend partly on the wrist and finger operation is good or bad, and the range depends on a knee and elbow.If want to have fast, relentless, quasi shooting action, the key lies in the knee bending angle and elbows, wrists, fingers action is coordinated.And continuous vertical jump is by the action of knee bending angle and elbow, wrist and finger coherent action, and shooting action is very similar to can said continuous vertical jumping to the opportunities presented by a similar test.

\section{Recommendations}

4.1 This research only focuses on the fixed point shooting as a test project, but it has a big gap in the situation of the fierce basketball match.It is suggested that the researchers in the future can move a shot as a test project, which is close to the game situation, and may have different results.

4.2 This study the penalty kick, ball and three-point shot point, are all facing the basket frame position, so the misses basket data distribution, more than before and after the placement of the left and right.Researchers in the future can be different locations (even at different points every time to do the shooting test, not only to do a fixed point of shooting) do shooting test, may be different results.

4.3 Push ups is a basketball player weight training as an important task, can increase upper body strength and shooting accuracy.The grass-roots and high level of basketball coaches and players, in addition to skills training to improve weight training, so that the next game, increase the antagonism of the player's body.

4.4 Two part of the ball, in the three training projects, the first test is better than the post test.The main two points ball shooting, the strength of the control and the body's coordination has considerable difficulty.It is recommended that the coaches should pay special attention to these two points when they are training the players to shoot two points.

\section{Reference}

[1] Wang Longfei, Liu Pei, Wang Quanchang. An analysis of the development of sports in the university sports [J]. Journal of Capital Institute of Physical Education, 2005 (6)

[2] Lu Guo, Li Dan. Physical self-concept and mental model of adolescents with different peer relations [J]. Journal of Changchun Normal University, 2006 (2)

[3] Yu Haitao, Chang Huili. In the teaching of physical education in Colleges and universities to carry out the feasibility of training analysis [J]. Journal of Henan Institute of Education Development: Natural Science Edition, 2005 (3)

[4] Zhai Qiang, Guo Dahai. Different fatigue degrees on the shooting hit rate of sensory ability [J]. Journal of Shenyang Institute of physical education, 2004 (4)

[5]Lu Liangqiu. On juvenile basketball players shooting hit rate of the main perception factors [J]. Guangzhou Institute of physical education, 2009 (5)

[6] Liu Ying. Analysis and pre control measures for the analysis of the dynamic damage of basketball players Chang Jianyun [J]. Liaoning sports science and technology, 2011 (1) 